\title{
Color stereopsis: A model for depth reversals based on border contrast
}

\author{
M. DENGLER and W. NITSCHKE \\ Luther College, Decorah, Iowa
}

\begin{abstract}
Color stereopsis refers to the effect of stimulus color on perceived depth of stimuli viewed binocularly. It is well established and well understood that the wavelength of a stimulus affects its perceived depth in color stereopsis by prismatic effects in human optics, with long-wavelength stimuli normally seen in front of short-wavelength stimuli. It is less well known, and not well understood, that the background of the stimulus affects depth order as well. We demonstrate experimentally, using a long-wavelength orange stimulus and a short-wavelength blue stimulus, that a change from black to white in the background at the border (a change in border contrast) of the stimulus changes the perceived depth order from orange in front of blue to blue in front of orange (a color reversal). We present a descriptive model to explain reversals in color stereopsis when stimuli are displayed on a white background and suggest that it may account for instances of color reversals that are currently unexplained or questionably explained by other models.
\end{abstract}

Many persons who have binocularly scrutinized colored maps, drawings, or photographs through a large magnifying lens have observed that the colors often appear to separate themselves into distinctly different depth planes. This phenomenon, referred to as color stereopsis or chromostereopsis (Benzschawel, 1985), was described over a century ago by Brewster (1851). He reported that, owing to the chromatic aberration of the lens, the longer wavelengths (reds, yellows) refract less than the shorter wavelengths (blues, greens) and thereby alter their retinal disparities. If a convex lens is used, short-wavelength colors are displaced nasally in comparison with longwavelength colors in each eye because of the prismatic distortion through each side of the lens, and they are seen stereoscopically as more distant.

Color stereopsis may also occur when color displays are viewed with unaided eyes (Donders, 1864, as cited by Hartridge, 1918; Einthoven, 1893; Luckiesh, 1918). This observation has been explained by the fact that the visual axis and optical axis of the eye are noncoincident. The visual axis, which is anchored at the fovea, typically passes through the nasal side of the pupil and cornea. The displacement of the center of the pupil from the visual axis produces prismatic deviations in such a way that a fixated white object would be separated into a small spectrum with the shorter wavelengths at the nasal side (Vos, 1960). Moreover, if equidistant red and blue stimuli were viewed, chromatic dispersion would displace blue more nasally on the retina than red in each eye, and we would

We thank J. R. Fletcher, University of Nottingham; Wayne Hershberger, Northern Illinois University; Jay McLaren, Mayo Clinic; and David T. Nelson, Luther College, for their help and useful discussion. Correspondence should be addressed to $W$. Nitschke, Department of Psychology, Luther College, Decorah, IA 52101. expect the observer to perceive a red-in-front-of-blue depth effect on the basis of the resulting binocular disparity (Vos, 1960; 1962). The observation of long-wavelength colors being seen in front of short-wavelength colors is referred to as positive color stereopsis.

Our interest in color stereopsis was prompted by reports of perceived depth orders that differed from those reported by Brewster (1851), which we refer to as color reversals. Explanations proposed for these reversals have implicated the pupil. One theory offers the possibility that variation in pupil centration in relation to the visual axis could produce reversals (Einthoven, 1893). Another theory suggests that reversals could occur if variation in pupil size altered the position of the effective pupil center as a result of the Stiles-Crawford effect (Vos, 1960, 1963, 1966). Our observations and those of our students and colleagues, carried out for several years with the use of numerous color displays, appeared to implicate another important variable. Border contrast, or the contrast in light intensity between color stimuli and light immediately adjacent to the stimuli, appears to be a critical contextual variable affecting color stereopsis depth orders in our displays.

In one series of observations, subjects binocularly viewed four colored threads-red, yellow, green, and blue-through a large convex lens when the colors were displayed on either a black or a white background. When the threads were displayed on a black background, almost all the subjects experienced positive color stereopsis with colors in the order given above. But when the threads were displayed on a white background, the same subjects reported, with rare exceptions, color reversals. Green was seen to be nearest the observer, followed by blue, red, and yellow. It should be noted that if the colored threads cast shadows on the white background, this change in background often led to positive color stereopsis. 
Our observations of color reversals are consistent with those reported earlier by Verhoeff (1928) and Hartridge (1947). Verhoeff noted that when viewed binocularly through a convex lens or by the unaided eye, blue letters appeared to be closer to the observer than red letters when the letters were written on white paper. Hartridge reported the following color order, from near to far when the colors were viewed binocularly, with unaided eyes, on a white background: blue-green, greenish blue, blue, purple, red, orange, and yellow. Neither Verhoeff nor Hartridge, however, provided experimental data in support of their observations.

The purposes of this paper are threefold. First, we offer experimental support for our observations and those of Verhoeff (1928) and Hartridge (1947) that, with the unaided eye, color stereopsis depth ordering is affected by background light intensity-more specifically, by border contrast. Second, we present a descriptive model to explain color reversals that result from border contrast. Finally, we propose that this model may account for some demonstrated color reversals that are now unexplained or accounted for by changes in pupil size.

In the following experiment, subjects made depth judgments without the aid of an external lens. They were shown computer-generated displays in which color stimuli were surrounded by light that was either darker or lighter than light from the stimuli themselves. The goal of the experiment was to demonstrate that border contrast alone could produce reversals in color stereopsis when color displays were viewed with the unaided eye.

\section{METHOD}

\section{Subjects}

Twenty-eight students from the general psychology class at Luther College served as subjects for extra class credit. Only students who reported having normal, uncorrected vision were selected to participate as subjects. Each subject passed a Dvorine Pseudoisochromatic Plates test of color perception and a test of stereopsis.

\section{Stimuli}

The subjects judged the perceived depth of orange and blue stimuli that were displayed in medium resolution on an Atari 1040ST computer. The colors orange and blue were selected from a palette of 512 colors because they are widely separated on the color spectrum and because their intensity levels were very similar on a Minolta Spotmeter M. When each color was displayed on a black screen, its intensity level was $3.92 \mathrm{~cd} / \mathrm{m}^{2}$. When blue was displayed on a white background, its intensity level was $3.17 \mathrm{~cd} / \mathrm{m}^{2}$ while that of orange was $2.77 \mathrm{~cd} / \mathrm{m}^{2}$. The intensity level of the white background measured $5.54 \mathrm{~cd} / \mathrm{m}^{2}$ while the black background produced no reading. For their intensity level measurements, the colors were displayed as $3 \times 3 \mathrm{~cm}$ squares on a black or white screen, which was masked by mat black cardboard.

Two different experimental display patterns were used: lines and boxes. The line pattern consisted of 14 alternating orange and blue lines. Each line was $54 \mathrm{~mm}$ in length and $1.4 \mathrm{~mm}$ in width, and it was separated by $2.8 \mathrm{~mm}$ from the neighboring lines. This pattern was presented for viewing on either a black or a white background. The box pattern consisted of fifteen $4.7 \times 5.8 \mathrm{~mm}$ blue or orange squares evenly spaced, 3 across and 5 down, on a background of the opposite color, either orange or blue. Each box was surrounded by a line that was $2.1 \mathrm{~mm}$ wide horizontally and $1.6 \mathrm{~mm}$ wide vertically. This line, which outlined the boxes and provided border contrast, was displayed as either black or white. The outlined boxes were separated by $3.5 \mathrm{~mm}$. The entire display measured $58 \times 47 \mathrm{~mm}$, and the computer monitor outside the display was black.

\section{Procedure}

The subjects were randomly assigned to either the light or the dark border contrast condition. A between-subject design was employed because of our concern that the results would be confounded by a perseveration response bias if a within-subject design were used. We expected that a subject's tendency to respond consistently under the two border contrast conditions could override the color stereopsis effect, which is weak for most subjects. In using this experimental design, we explicitly assumed that for a vast majority of subjects there is a noncoincidence of the visual axis and optical axis, with the visual axis passing through the nasal side of the pupil and cornea (Vos, 1960).

In the dark border contrast condition, the subjects viewed the lines on a black background and viewed boxes that were outlined in black. In the light border contrast condition, the subjects viewed the lines on a white background and viewed boxes that were outlined in white.

The subjects within a condition first judged the lines and then the boxes twice, blue on orange followed by orange on blue (or the reverse order for half the subjects); then they judged the lines a second time. There were 14 subjects in each condition. All judgments were made in a dark room illuminated only by a $25-\mathrm{W}$ incandescent lamp placed out of the subjects' line of sight and by the computer monitor itself. The subjects were seated so that their eyes were approximately $1 \mathrm{~m}$ from the monitor, but they were free to move back or forward if they judged that such movement improved their viewing.

Each subject was told that the purpose of the experiment was to assess whether the color of a figure affected its perceived distance from the observer or from other nearby color figures. Each was told that people sometimes report that a red or a green or some other colored object appears to be nearer to them, or to rise above different nearby colors, and that at other times it seems to fall behind, or is seen as farther away than the other colors. Each subject was instructed to view the displays for about $30 \mathrm{sec}$, or as long as was necessary for them to decide whether one of the colors appeared to be in front of, or in back of, the second color. At the end of the viewing period, the subjects were asked whether one of the colors appeared to be nearer to them or to rise above the other color, or whether the display was essentially flat with no difference in depth between the two colors

\section{RESULTS AND DISCUSSION}

The subjects were scored as seeing blue in front of orange, or blue in back of orange, or as seeing no depth if they were consistent in all of their judgments on the two presentations of each pattern. Otherwise, they were scored as inconsistent. Responses for the line and box patterns were scored separately.

The experimental results are presented in Figure 1. The no-depth and inconsistent judgments were both regarded as providing no evidence for stereoscopic depth perception and were combined for this graph. It can be seen that the modal depth judgment for the two patterns under the 


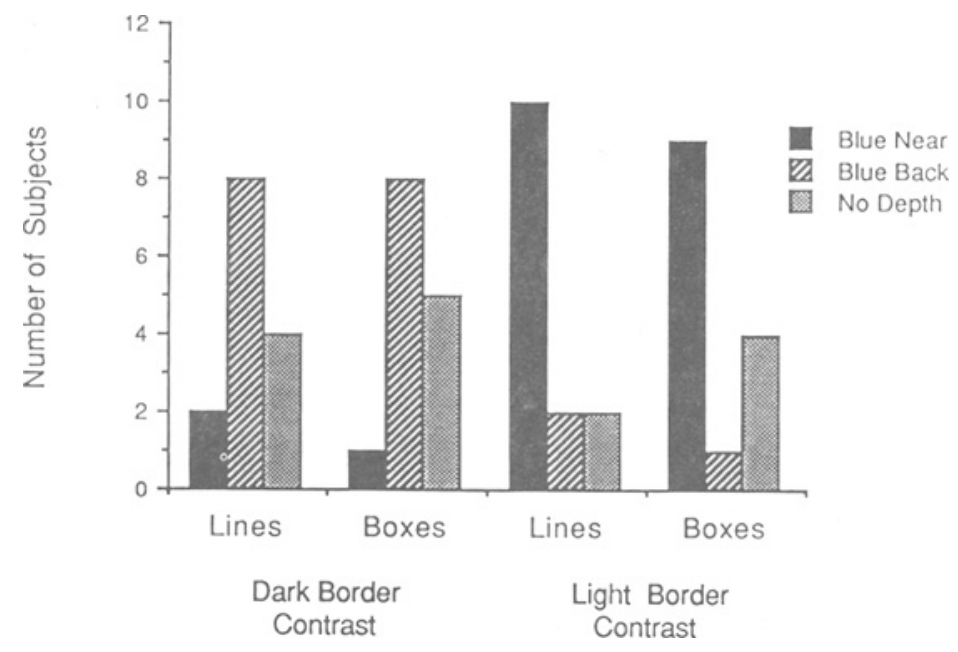

Figure 1. The number of subjects who judged blue in comparison with orange to be nearer, farther back, or with no depth, for two different blue-orange patterns and two different border contrast conditions-dark or light.

dark border contrast condition consisted of blue in back of orange. This judgment reversed, and the modal depth response consisted of blue in front of orange for the light border contrast condition.

The differences in the frequency of the depth judgments, blue near or blue back, were analyzed for statistical significance with a binomial test. For the dark border contrast group, the two-tailed $p$ values were .1094 for the lines pattern and .0201 for the boxes pattern. For the light border contrast group, the two-tailed $p$ values were .0296 for the lines and .0290 for the boxes.

The pattern of experimental results was consistent with our previous observations and with those of Verhoeff (1928) and Hartridge (1947); it demonstrated the effect of border contrast on color stereopsis. The shortwavelength color, blue, was seen in back of the longer wavelength color, orange, when the intensity level of these stimuli was greater than that of the light immediately surrounding the stimulus. This depth order reversed, however, when the intensity of the surrounding white light was greater than the stimulus intensity.

\section{OPTICAL SHIFT MODEL OF COLOR REVERSALS BASED ON BORDER CONTRAST}

We propose that color stereopsis depth reversals are attributable to two factors: the wavelengths of both stimulus and background light, and border contrast. When the background light at the stimulus border is comparatively more intense than the stimulus light, the light rays from the two surfaces refract differentially to yield an optical shift. But when the background light is comparatively less intense than the stimulus light, the stimulus wavelengths refract without being affected by the refracted background light. In Figure 2, we depict, in a schematic representa- tion, how these shifts occur for four different color stimuli, each of which is displayed on a white background.

In Figure 2, (A) depicts the stimulus as less intense than the background. Each quadrant of the figure depicts one of four different stimuli. In (B), we show the prismatic nasal shift of the stimulus on the retina that is produced by the noncoincidence of the visual and optical axes. A comparison of the four different-colored stimuli depicted also shows an increase in shift with a decrease in wavelength. In (C), we show the prismatic nasal shift of the polychromatic white background light into a diagrammatic spectrum of red, yellow, green, and blue, with each color shown independently. Note that the various colors break out differentially, depending on wavelength, and that no color $(0)$ occurs for each background wavelength, owing to the spatial position of the stimulus. This representation is identical for each of the four stimuli shown. In (D), the stimulus previously shown in (B) is redrawn and modified to show how the background light produces two optical effects, fringe attachment and whiting out, which, we propose, alter the lateral retinal displacement of the refracted color stimuli and lead to stereoscopic color reversals. Fringe attachment refers to the incorporation of a like-colored fringe to a perceived color stimulus, thus lengthening the perceived color stimulus in the direction of the incorporated color fringe. We represent fringe attachment as a graded effect beginning with the addition of background wavelengths to the stimulus and ending where the incorporated wavelengths produce polychromatic white. (D) shows that the attachment of a fringe could only occur on the nasal side of the retina for red and yellow and on the temporal side for green and blue. It can also be seen that attached fringes for red and blue stimuli begin with wavelengths similar to that of the stimulus. However, in the case of yellow and green stimuli, background light first changes the chromaticity of the 


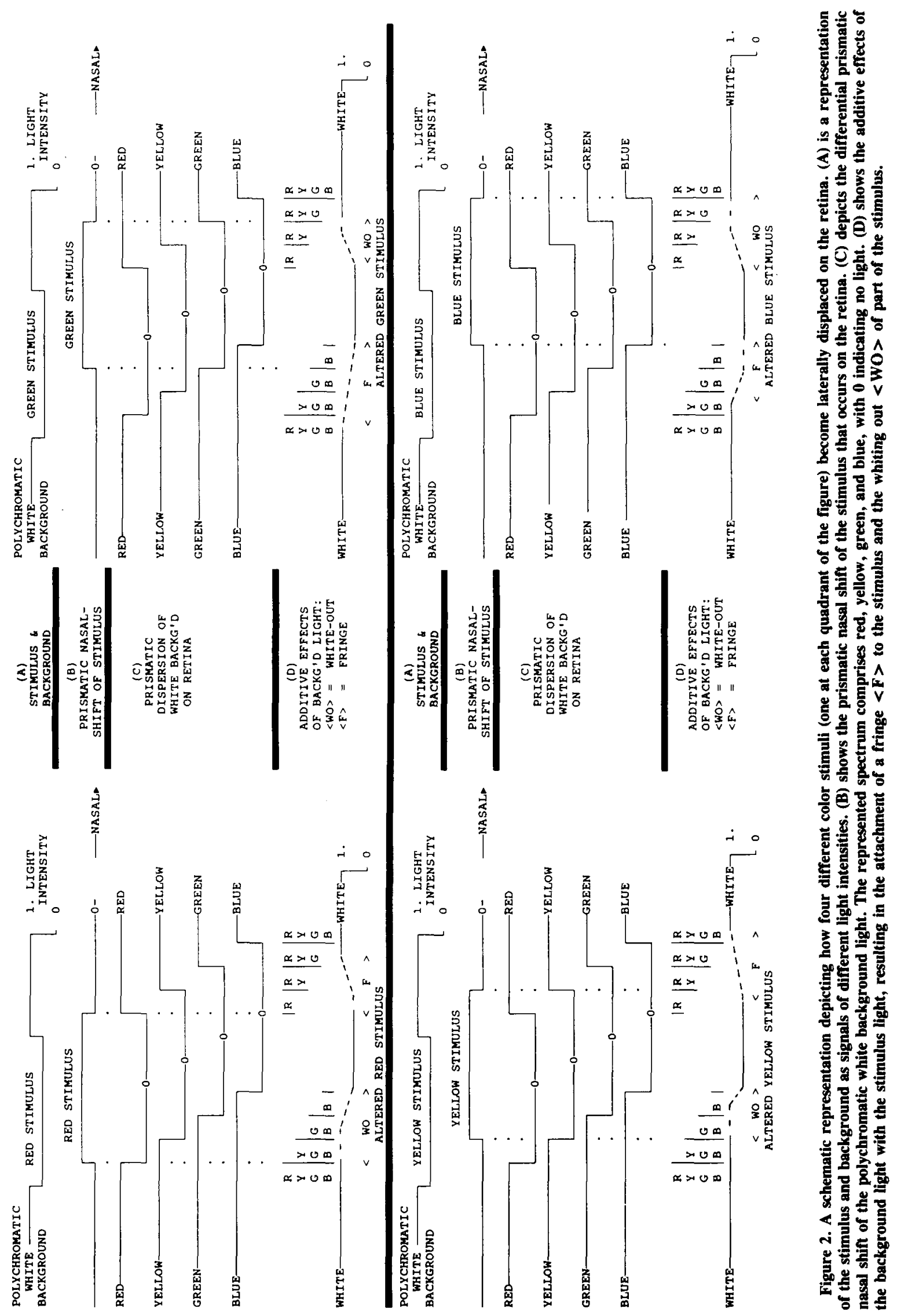




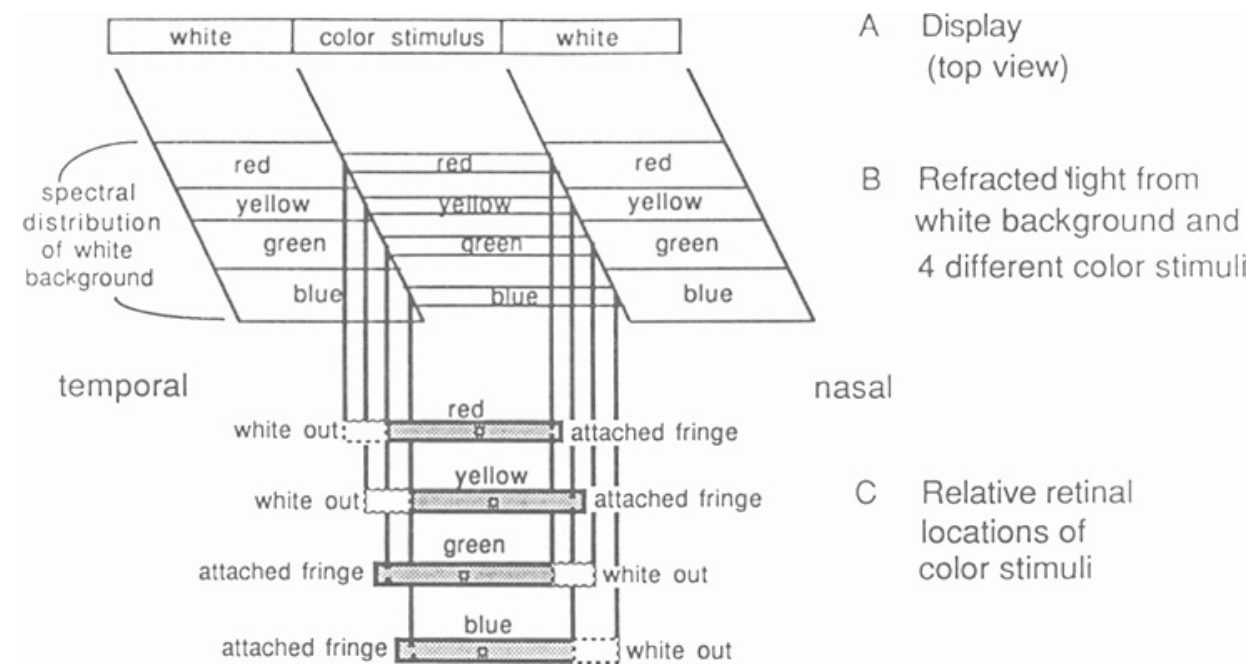

\begin{abstract}
Figure 3. A composite model for the perception of color reversals. (A) A top view of a display containing a color stimulus placed on a polychromatic white background. (B) The continuous change of the refracted spectral distribution of the white background in addition to how four spectral-specific stimuli would refract. (C) The proposed optical effects of fringe attachment and whiting out alter the lateral retinal location of the four refracted color stimuli. The altered stimuli are shown as a filled rectangle with a new lateral displacement on the retina marked at the stimulus midpoint $(\square)$.
\end{abstract}

stimulus at one edge before similar wavelengths are attached.

Whiting out occurs when overlapping wavelengths create additive color mixture and effectively reduce color saturation until white is perceived at one edge of each stimulus. Diagrammatically, we represent the extent of whiting out in Figure 2 (D) also as a graded effect, beginning with the addition of new background wavelengths to the stimulus and culminating at the point where the background and stimulus light are complementary. Using this principle, the model shows whiting out of the stimulus occurring on the temporal side of the retina for red and yellow and on the nasal side for green and blue.

The principles of whiting out and fringe attachment shown separately for each color stimulus in Figure 2 are also shown in a composite diagram in Figure 3. This depiction more clearly represents the continuity of wavelengths in the background light, and it shows how the spectral energy of the stimuli appear in that context. It can be seen here that the most temporally displaced stimulus is green and the most nasally displaced stimulus is yellow. Our model also shows that, for the depicted red and blue stimuli, the lateral displacement on the retina is approximately equal, and therefore these colors would be perceived to be near to one another in stereoscopic depth when displayed on a white background. The model shows the complete four-colored stereoscopic color reversal order to consist of green closest to the observer, followed by blue/red and yellow, which is consistent with our earlier observations and those of Hartridge (1947).

Our physical evidence for the effect of fringe attachment and of whiting out was obtained in a series of black and white photographs of a stimulus display taken through a positive refractive lens approximately along the path of vision for the left eye. The display consisted of four different-colored threads (red, yellow, green, blue) placed in front of a background in which the top half was black and the bottom half was white. Two of the photographs are shown in Figure 4, with the red thread appearing at the left and the green thread at the right. Inspection of the photographs shows that the borders of the threads shift as the threads cross backgrounds.

Our examination of all the photographs showed that these border shifts correspond to the chromatic changes associated with the process of fringe attachment and whiting out. Fringe attachment is seen for threads on a white background, since their borders extend beyond the borders of the same thread on a black background. The whiting out effect is seen, since the borders of the threads on a white background appear to be displaced inwardly, relative to the borders of the same threads as they appear on the dark background.

For red and yellow threads on a white background, both fringe attachment and whiting out shift borders outwardly from the lens center. This displacement produces a nasal shift on the retina and results in the perception that the threads recede from the observer on a white background. The borders of green and blue threads appear to be displaced inwardly toward the center of the lens on a white background, which produces a temporal shift on the retina and results in the perception that they are nearer to the observer.

\section{MODEL IMPLICATIONS}

There is a long history of accounting for stereoscopic color reversals in terms of changes in pupil size. Einthoven (1893) recognized that if the center of the pupil 


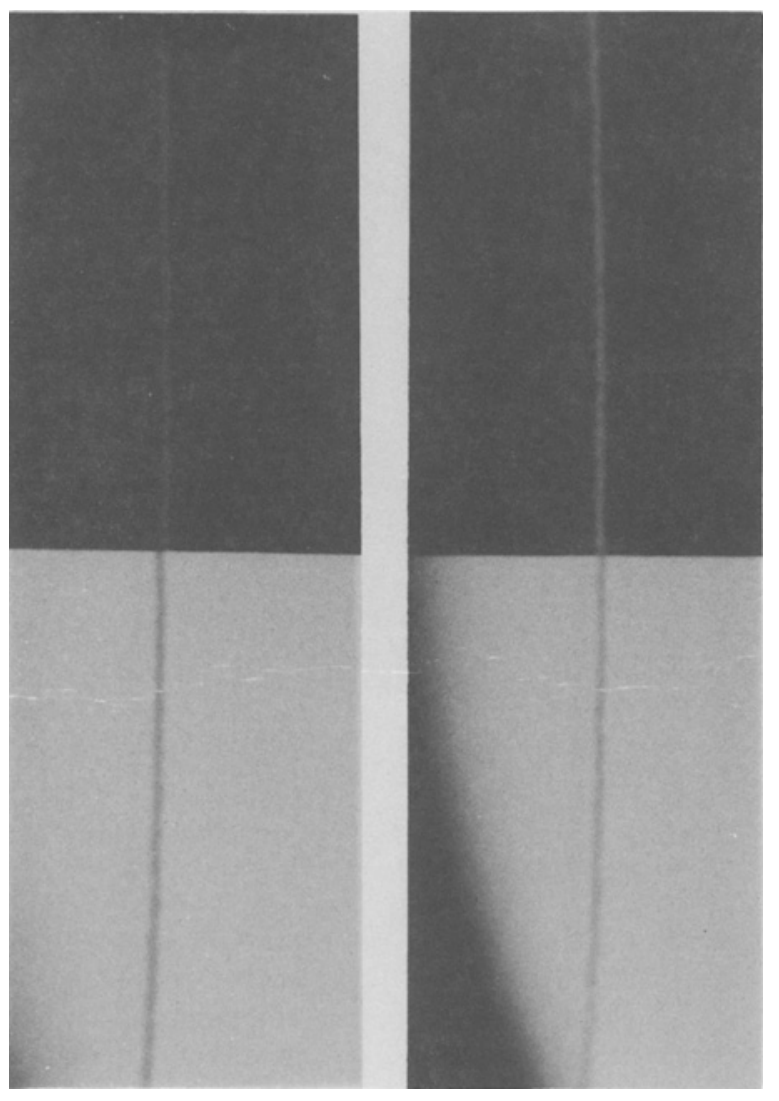

Figure 4. Photographs taken through a positive refractive surface and approximately along the path of vision of the left eye of a red (left) and a green (right) thread. Each thread, as it crosses in front of the white background, appears to shift: the red thread shifts left or nasally, and the green thread shifts right or temporally. See text for discussion of attached fringes and whiting out.

shifted across the visual axis as it opened, a color reversal would be predicted. Indeed, evidence that changes in pupil centration do occur has been provided by Walsh (1988), but the reported magnitudes are quite small. Vos $(1960,1963,1966)$ on the other hand, suggested the interesting possibility that with changes in pupil size the effective center of the pupil might shift because of the Stiles-Crawford effect. He noted (Vos, 1966) that the Stiles-Crawford effect is almost eliminated with small pupil size, suggesting that color reversals are contingent upon large pupil size. Sundet (1972), who made observations on himself and 2 students under conditions in which the students' pupils were pharmacologically contracted and dilated, provided data in support Vos's theory. He reported that reversals were obtained when the pupil was dilated.

Simonet and Campbell (1990), using 30 subjects and varying pupil size by varying ambient illumination, reported that only 5 of 30 subjects observed reversals in the direction predicted by Vos's theory. Furthermore, these investigators noted that the magnitude of changes in pupil size was not related to color reversals, since the magnitude of change was identical when no reversal occurred. In this study, therefore, Vos's theory did not account for color reversals that were produced under natural viewing conditions, where pupil size was not pharmacologically manipulated as it was in Sundet's study.

Using our model, we would have predicted that subjects in the experiment by Simonet and Campbell (1990) would have reported red-in-front-of-blue positive color stereopsis, with no reversals occurring because the stimuli were displayed only on a black background. Simonet and Campbell reported that under each pupil condition approximately $50 \%$ of the subjects reported positive color stereopsis. We suggest that more would have made that observation if it had not been for the orientation of the stimuli. The display for stereoscopic depth comparisons always presented a red stimulus positioned directly above a blue stimulus. The display, therefore, contained a second cue to depth-relative height, which directly opposed positive color stereopsis.

Kishto (1965), in his influential work, reported positive color stereopsis under high levels of illumination and color reversals under low levels of illumination, a finding in apparent support of Vos's theory and in apparent disagreement with our model. We believe, however, that levels of illumination and border contrast may have been confounded in Kishto's study and that his experimental results are equivocal. Kishto's "best estimates" of illumination values showed overlapping ranges for stimuli and background. Border contrast values, therefore, may have occasionally satisfied the necessary condition for color reversals in our model-that is, light border contrast. We agree with Simonet and Campbell (1990) that the levels of illumination under which Kishto performed his measurements were poorly controlled. Equally important, we suggest, is that Kishto's apparatus might have easily introduced spurious border contrast effects. His color stimuli were cut patches made from stiff paper or card. It has been our experience in designing various stimulus displays that cut edges are difficult to conceal and that the reflected light from the edges may serve as border contrast. Furthermore, because Kishto's apparatus consisted of a slide mechanism with stimuli positioned in front of a white screen, there is a distinct possibility of shadows' having been cast on the background and altering the border contrast. Recall our earlier observation, which guided the design of our displays. The introduction of shadows with colored threads frequently changes border contrast; or, for that matter, merely drawing a thin line around a color stimulus printed on a white surface reverts the color reversal to positive color stereopsis.

In conclusion, it appears that explanations of color reversals based on light-intensity-induced changes in pupil size have not received strong empirical support. It is also apparent that much of the previous research on color stereopsis may have unintentionally confounded border contrast with illumination levels, an inherent problem of three-dimensional displays. We conjecture that border 
contrast may have influenced color reversals in earlier work, but since it was not experimentally controlled, its effect was obscured.

\section{REFERENCES}

Benzschawel, T. (1985, September). Human factors influence effective use of color in information displays. Information Display, pp. 16-34.

BREWSTER, D. (1851). Notice of a chromatic stereoscope. Philosophical Magazine, 4th Series, 3, 31

EINTHOVEN, W. (1893). On the prediction of shadow and perspective effects by difference of colour. Brain, 16, 191-202.

HARTRIDGE, H. (1918). Chromatic aberration and resolving power of the eye. Journal of Physiology, 52, 175-246.

Hartridge, H. (1947). The visual perception of fine detail. Philosophical Transactions of the Royal Society, 232, 519-671.

Kishto, B. N. (1965). Colour stereoscopic effect. Vision Research, 5, 313-329.

LUCKIESH, M. (1918). On "retiring" and "advancing" colors. American Journal of Psychology, 29, 182-186.
Simonet, P., \& Camprell, M. C. W. (1990). Effect of illuminance on the directions of chromostereopsis and transverse chromatic aberration observed with natural pupils. Ophthalmic \& Physiological Optics, 10, 271-279.

SUNDET, J. M. (1972). The effect of pupil size variations on the colour stereoscopic phenomenon. Vision Research, 12, 1027-1032.

VERHOEFF, F. H. (1928). An optical illusion due to chromatic aberration. American Journal of Ophthalmology, 11, 898-900.

Vos, J. J. (1960). Some new aspects of color stereoscopy. Journal of the Optical Society of America, 50, 785-790.

Vos, J. J. (1963). An antagonistic effect in colour stereoscopy. Ophthalmologica, 145, 442-445.

Vos. J. J. (1966). The color stereoscopic effect. Vision Research, 6 , 105-107.

WAISH, G. (1988). The effect of mydriasis on the pupillary centration of the human eye. Ophthalmic \& Physiological Optics, 8, 178-182.

(Manuscript received April 10, 1991; revision accepted for publication July 28, 1992.) 